\title{
Phytodiversity (Angiosperms and Gymnosperms) in Chaurangikhal forest of Garhwal Himalaya, Uttarakhand, India
}

\author{
Sarvesh Suyal, C.M. Sharma, Sumeet Gairola, S.K. Ghildiyal, C.S. Rana and D.S. Butola \\ Dept. of Botany, HNB Garhwal University, Srinagar Garhwal-246 174, Uttarakhand, India. \\ sharmacmin@gmail.com; sarveshsuyal@gmail.com
}

\begin{abstract}
We report the phytodiversity richness of the moist temperate Chaurangikhal forest of Garhwal Himalaya, Uttarakhand, India. We recorded a total of 231 species (227 angiosperms and 4 gymnosperms) belonging to 69 families (67 angiosperms and 2 gymnosperms) and 159 genera (156 angiosperms and 3 gymnosperms). The dicotyledones and monocotyledones were represented by a total of 62 and 5 families, respectively. In the study area, the ratio of family to genus was $1: 2.3$, family to species was $1: 3.35$ and a genus to species was $1: 1.45$. Among all the species recorded the $88.31 \%$ (204 spp.) of the total species had common occurrence, whereas rest $11.69 \%$ (27 spp.) of the species had uncommon occurrence in the study area. The 10 dominant families of the study area were Lamiaceae, Asteraceae, Rosaceae, Ranunculaceae, Fabaceae, Caryophyllaceae, Polygonaceae, Rubiaceae, Gentianaceae and Poaceae. This data may be useful for biodiversity managers and for optimal utilization of plant resources.
\end{abstract}

Keywords: India, phytodiversity, floristic composition, plant distribution, Garhwal Himalaya, moist temperate forest.

Introduction

Vegetation is the most precious gift, nature has provided to us, as it is meeting all kinds of essential requirements of the humans in the form of food, fodder, fuel, medicine, timber, resins, and oil, etc. (Gaur, 1999). Plant community plays a pivotal role in sustainable management by maintaining biodiversity and conserving the environment (Farooquee \& Saxena, 1996). The knowledge of the floristic composition of a plant community is a prerequisite to understand the overall structure and function of any ecosystem. The living world is composed of more or less distinct entities which are called species. They represent an important level of integration in living nature (Venu, 2002). Fundamental botanical research revolves around floristic (listing of all plants of a given area) and mono-graphic (study of a plant group for its entire range of distribution) works. A flora enumerates plants of a particular geographical area with a purpose to identify them (Venu, 2002).

The Indian subcontinent, with its rich biodiversity, is one of the 12 mega-diversity countries in the world. The Eastern Ghats, Western Ghats, Himalayas, North-eastern hills and Andamans constitute important biodiversity areas of India. Montane Himalayan forests are the most biologically diverse habitats. The Indian Himalayan region occupies a special place in the mountain ecosystems of the world. These geodynamically
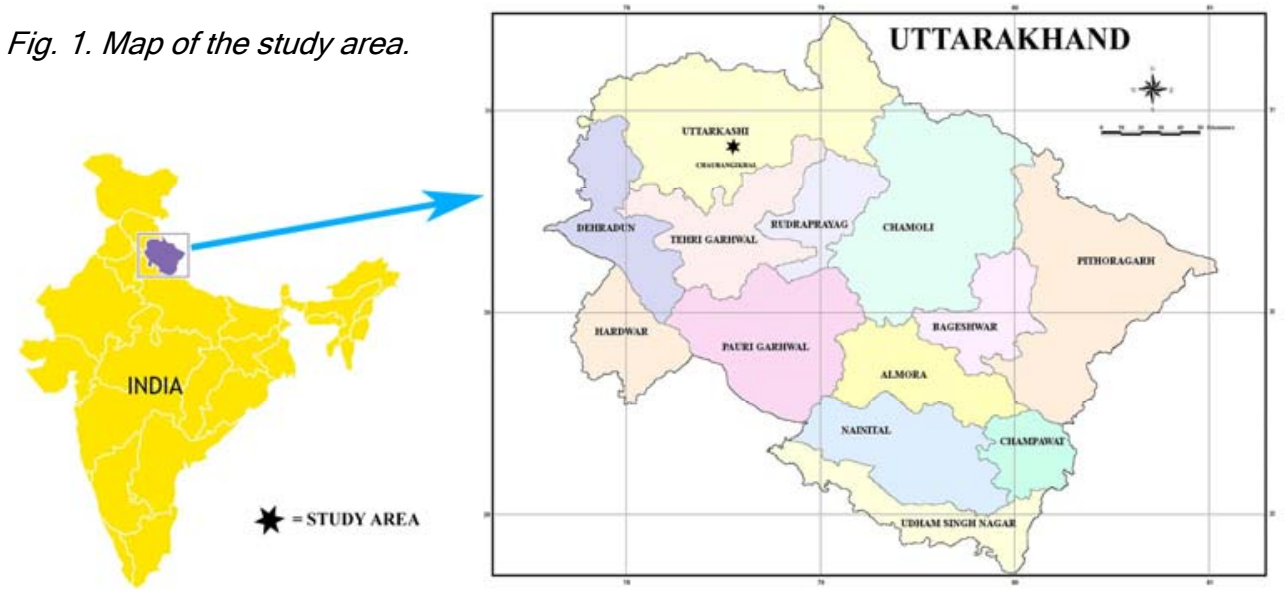

"Phytodiversity of Garhwal Himalaya forest" http://www.indjst.org young mountains are not only important from the stand point of climate and as a provider of life, giving water to a large part of the Indian subcontinent, but they also harbour a rich variety of flora, fauna, human communities and cultural diversity (Singh, 2006). The Himalayan mountain system covers only $18 \%$ of the geographical area of India, but accounts for more than $50 \%$ of India's forest cover and for $40 \%$ of the species endemic to the Indian subcontinent. About $64 \%$ of total geographical area of Uttarakhand is covered with forest (FSI, 2003). The fascinating flora of Northwest Himalaya with different topographic and climatic zones has attracted attention of several professional botanists, environmentalists and people of other walks viz. medical practitioners, engineers, surveyors, foresters, naturalists, etc. The Northwest Himalaya has long been recognized as a distinct floristic region in India (Hooker, 1906). Owing to varied topography, wide altitudinal range and unique 


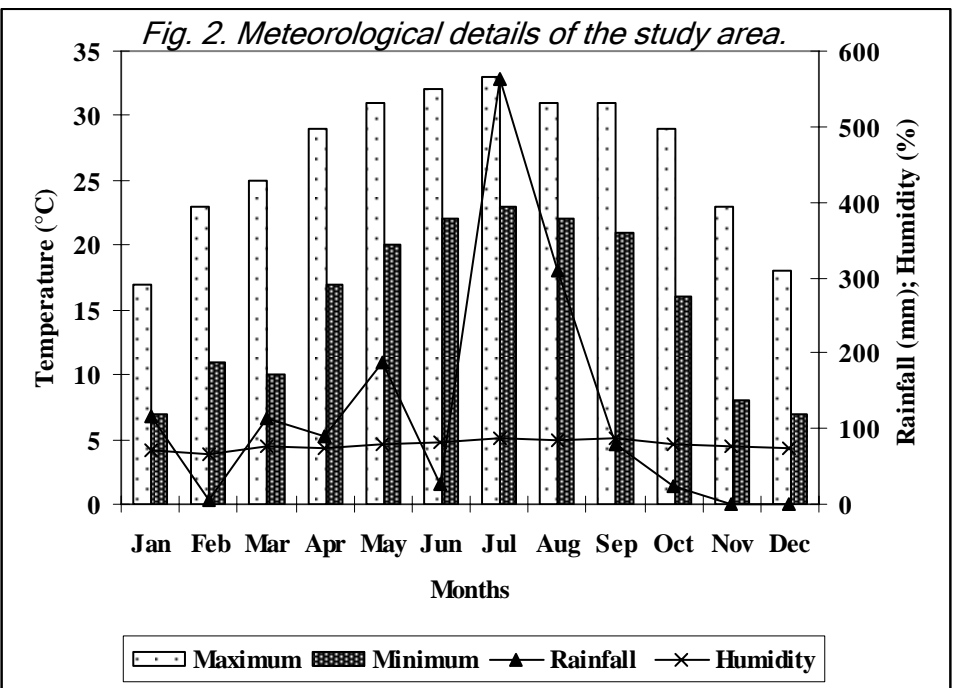

geographical location, this region harbours a rich flora and exhibits affinities with the Mediterranean, Siberian,

Fig. 3. Distribution of different groups according to taxonomic cateqories.

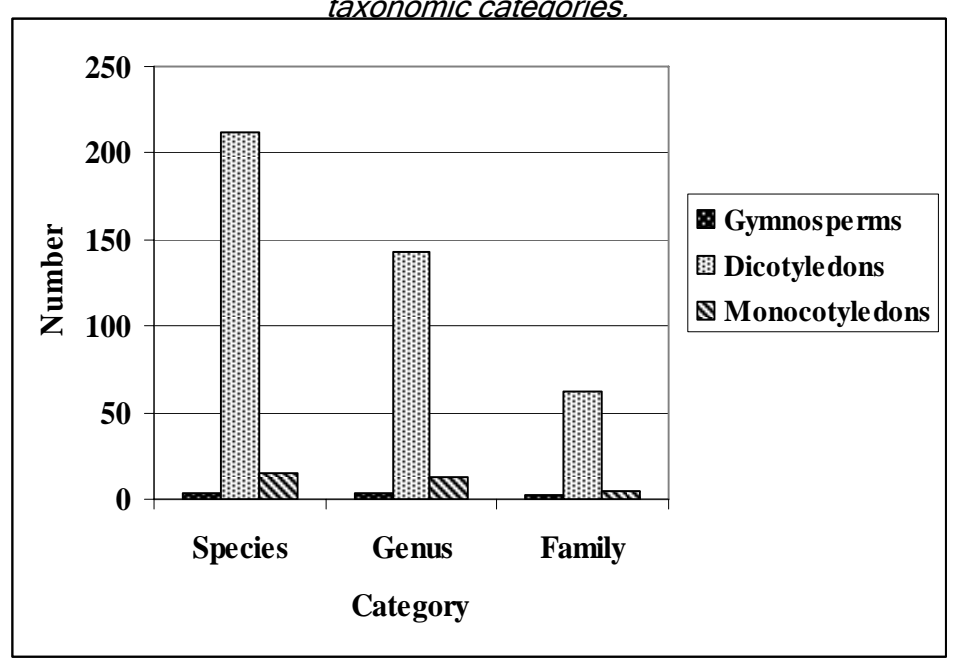

Tibetan and Indo-Malayan regions. Paleobotanical evidences indicate that many of the woody elements in the flora of the Himalayan region owe their origin from the tropical wet evergreen climate of the Indian peninsula. The Indian Himalayan region is considered as the repository of biological and cultural diversity and supports about 18,440 species of plant, including 1748 species of medicinal plants and 675 species of wild edibles (Negi \& Gaur, 1994). The representative biodiversity rich areas of the IHR have been protected through a protected area network (PAN). At present there are 5 biosphere reserves, 28 national parks, and 98 wildlife sanctuaries in IHR covering $51,899.238 \mathrm{~km}^{2}$ (Mathur et al., 2000). These protected areas are distributed in tropical, temperate, and alpine Himalayan ecosystems.

The concern grew on paramount after the constitution of "World Commission on Environment and Development (WCED) by the general assembly of the UN. This endorsed the need of conserving the world's rich
Vol. 3 No. 3 (Mar 2010)

ISSN: 0974- 6846

biodiversity particularly that of the tropical areas. The "Earth Summit" a global convention held at Rio De Janerio (1992) under the auspices of United Nations Commission on Environment and Development (UNCED) laid stress on the integration, conservation and sustainable use of biodiversity. The biodiversity convention was ratified by 104 countries by adopting 42 articles as future course of action. One of the prerequisite tasks expressed by article 7 of the convention is the "identification and monitoring the components of biological diversity" while article 12 calls for research and training and suggests programme for identification, conservation and sustainable use of biological diversity. Documenting basic patterns of biodiversity is fundamental for priotizing areas for conservation and management action (Villasenor et al., 2007). Recently study on forest composition, diversity and socio-economic status in the Garhwal and study area has been done by Sharma \& Gairola, 2007; Gairola et al., 2009a,b; Sharma et al., 2009a,b,c; Sharma et al., 2010. The Chaurangikhal forest is very rich in plant diversity and enumeration of plant wealth of this area has not been done so far. Therefore, in this study we have tried to list the flora of this forest covering all the important aspects of the phytodiversity (angiosperms and gymnosperms) of the study area.

\section{Study area}

Chaurangikhal forest is a part of the Mukhem forest division in Uttarkashi district of Uttarakhand. Its main station, Chaurangikhal is situated $29 \mathrm{Km}$ away from Uttarkashi town. The temples of Chaurangi Nath Baba and Nachiketa Bhagwan are well known tourist attraction of the area. The forest area covers an altitudinal range of $1650 \mathrm{~m}$ asl to $2800 \mathrm{~m}$ asl and is situated at latitude $30^{\circ} 39.125^{\prime} \mathrm{N}$ and longitude $78^{\circ}$ 31.156' E (Fig. 1). Meteorological details of the study area are shown in Fig. 2. At Chaurangikhal station (situated at $2310 \mathrm{~m}$ asl), nearly $80 \%$ of the mean annual rainfall $(1516 \mathrm{~mm})$ in year 2006-07 occurred in the monsoon season between June and September and 20\% fells as snow in winter season, between December and March. In nearby Chaundiyar and Dikholi villages (at $1650 \mathrm{~m}$ asl), the temperature remains cool and pleasant round the year. Frost is common during winter season, while the higher elevations (Harunta Bugyal at $2700 \mathrm{~m}$ asl and Thang area at $2550 \mathrm{~m}$ asl) experience heavy spells of snowfall, which may persist up to April-May in shady locations. Mean minimum monthly temperature ranged from $7.12^{\circ} \mathrm{C}$ (Jan) to $23.20^{\circ} \mathrm{C}$ (Jul) and mean maximum monthly temperature ranged from $17.56^{\circ} \mathrm{C}$ (Jan) to $33.35^{\circ} \mathrm{C}$ (Jul) in the year 2007.

\section{Methodology}

Extensive field surveys were conducted in the study area from May 2006 to April 2009 in different seasons (rainy, winter and summer) to assess the diversity of higher plants (gymnosperms and angiosperms) including
Research article

CIndian Society for Education and Environment (iSee)
"Phytodiversity of Garhwal Himalaya forest" http://www.indjst.org
Sarvesh Suyal et al. Indian J.Sci.Technol. 
Vol. 3 No. 3 (Mar 2010)

ISSN: 0974- 6846

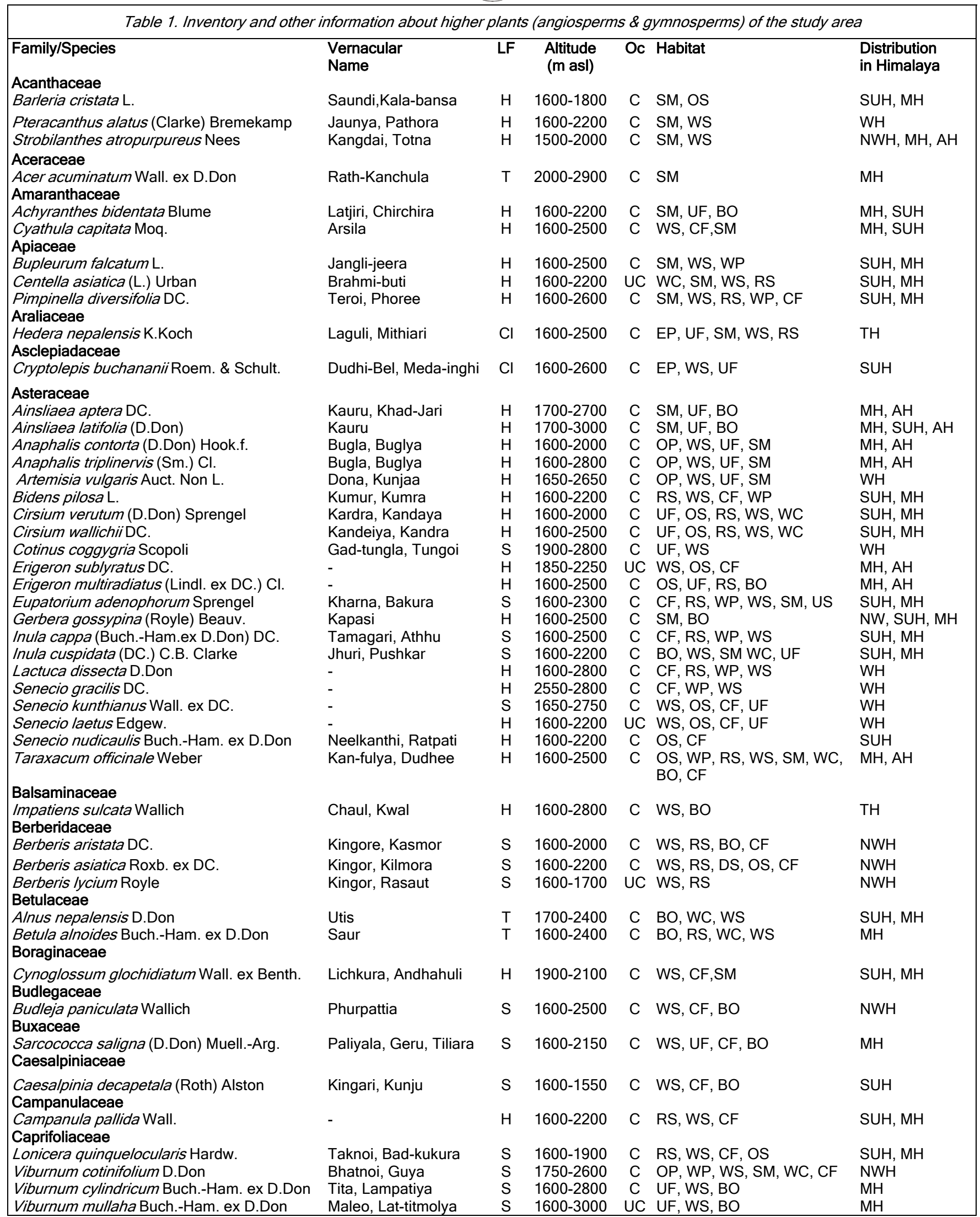

Research article

CIndian Society for Education and Environment (iSee)
"Phytodiversity of Garhwal Himalaya forest" http://www.indjst.org
Sarvesh Suyal et al. Indian J.Sci.Technol. 


\begin{tabular}{|c|c|c|c|c|c|c|}
\hline Caryophyllaceae & (Table 1 continues) & & & & & \\
\hline Cerastium cerasoides $\mathrm{L}$. & - & $\mathrm{H}$ & $2450-2650$ & UC & CF, WS, RS, UF, SM & $\mathrm{MH}, \mathrm{AH}$ \\
\hline Cerastium glomeratum Thuillier & - & $\mathrm{H}$ & $1600-3000$ & $\mathrm{C}$ & CF, WS, RS, UF, SM & $\mathrm{MH}, \mathrm{AH}$ \\
\hline Gypsophylla cerasoides D.Don & Bakarchee & $\mathrm{H}$ & $1600-2700$ & $\mathrm{C}$ & BO, WS, SM WC, UF & $\mathrm{TH}$ \\
\hline Sagina saginoides (L.) Karsten & - & $\mathrm{H}$ & $2300-2700$ & C & WS, UF, CF, BO & $\mathrm{TH}$ \\
\hline Silene edgeworthii Bocquet & Bakrolya & $\mathrm{H}$ & $1600-2000$ & UC & WS, OS, CF, UF & $\mathrm{MH}, \mathrm{AH}$ \\
\hline Silene indica Roxb. ex Otth & - & $\mathrm{H}$ & $2400-2700$ & UC & OS, W, CF & $\mathrm{TH}$ \\
\hline Stellaria media (L.) Vill. & Badyalu & $\mathrm{H}$ & $1500-3000$ & $\mathrm{C}$ & WC, CF, WS & SUH \\
\hline $\begin{array}{l}\text { Stellaria monosperma D.Don } \\
\text { Chenopodiaceae }\end{array}$ & - & $\mathrm{H}$ & $1600-2600$ & $\mathrm{C}$ & WS, OS, CF, UF, RS & SUH \\
\hline $\begin{array}{l}\text { Chenopodium album } \mathrm{L} \text {. } \\
\text { Coriariaceae }\end{array}$ & Bathua, Bathu & $\mathrm{H}$ & $1600-2500$ & $\mathrm{C}$ & UF, OS, WS, WC & $\mathrm{TH}$ \\
\hline $\begin{array}{l}\text { Coriaria nepalensis Wallich } \\
\text { Cornaceae }\end{array}$ & Makroli, Gangara & S & $1600-2800$ & UC & UF, OS, WS, WC & $\mathrm{TH}$ \\
\hline Benthamidia capitata (Wallich ex Roxb.) Hara & Bhamora, Therbal & $\mathrm{T}$ & $1600-2500$ & $\mathrm{C}$ & SM, UF, BO & $\mathrm{MH}$ \\
\hline $\begin{array}{l}\text { Swida oblonga (Wall.) Sojak } \\
\text { Corylaceae }\end{array}$ & Katkanai & $\mathrm{T}$ & $1500-3000$ & UC & WS, UF, WC, SM & $\mathrm{MH}$ \\
\hline $\begin{array}{l}\text { Carpinus viminea Lindl } \\
\text { Crassulaceae }\end{array}$ & Chamkharik & $\mathrm{T}$ & $1600-2000$ & C & UF, RS, SM & $\mathrm{MH}, \mathrm{SAH}$ \\
\hline $\begin{array}{l}\text { Sedum oreades (Decne.) Hamet } \\
\text { Daphniphyllaceae }\end{array}$ & - & $\mathrm{H}$ & $1800-2800$ & $\mathrm{C}$ & WS, CF, UF, SM & $\mathrm{MH}, \mathrm{SAH}$ \\
\hline $\begin{array}{l}\text { Daphniphyllum himalayense (Benth.) Muell.- } \\
\text { Arg. }\end{array}$ & Ratniyalu & $\mathrm{T}$ & $1800-2500$ & $\mathrm{C}$ & UF, SM, RS & $\mathrm{NWH}$ \\
\hline Dioscoreaceae & & & & & & \\
\hline Dioscorea deltoidea Wallich ex Grisebach & Harvish, Tarur & $\mathrm{H}$ & $2350-2700$ & UC & WS, OS, CF & $\mathrm{MH}, \mathrm{AH}$ \\
\hline $\begin{array}{l}\text { Dioscorea melanophyma Prain \& Burkill } \\
\text { Dipsacaceae }\end{array}$ & Mangaii & $\mathrm{H}$ & $1600-2800$ & UC & UF, WS, CF & SUH \\
\hline $\begin{array}{l}\text { Dipsacus inermis Wallich } \\
\text { Ericaceae }\end{array}$ & Phulee & $\mathrm{H}$ & $1900-2500$ & $\mathrm{C}$ & WS, OS, CF & $\mathrm{MH}, \mathrm{AH}$ \\
\hline Gaultheria nummularioides D.Don & Bhwinla & $\mathrm{H}$ & $1600-2700$ & $\mathrm{C}$ & $\mathrm{RS}, \mathrm{UF}, \mathrm{BO}, \mathrm{OS}$ & $\mathrm{MH}, \mathrm{AH}$ \\
\hline Lyonia ovalifolia (Wall.) Drude & Anyar & $\mathrm{T}$ & $1600-2500$ & $\mathrm{C}$ & SM, OS, WS, CF, RS, UF & $\mathrm{MH}$ \\
\hline $\begin{array}{l}\text { Rhododendron arboreum Sm. } \\
\text { Euphorbiaceae }\end{array}$ & Burans & $\mathrm{T}$ & $1600-2300$ & $\mathrm{C}$ & $\mathrm{SM}, \mathrm{RS}, \mathrm{WP}, \mathrm{CF}$ & $\mathrm{MH}$ \\
\hline Euphorbia hirta L. & Dudhi & $\mathrm{H}$ & $1600-2300$ & $\mathrm{C}$ & CF, RS, WP, WS & $\mathrm{TH}$ \\
\hline Euphorbia pilosa L. & Chuplya, Chounpalu & $\mathrm{H}$ & $1850-2600$ & $\mathrm{C}$ & RS, WP, CF & $\mathrm{WH}$ \\
\hline $\begin{array}{l}\text { Excoecaria acerifolia F. Didr. } \\
\text { Fabaceae }\end{array}$ & Dudhila, Phutkiya & S & $1600-2500$ & C & SM, RS, WP, CF & $\mathrm{WH}, \mathrm{CNH}$ \\
\hline Crotalaria medicaginea Buch.- Hem.ex Roxb. & Ban methi, Gulabi & $\mathrm{H}$ & $1600-2500$ & $\mathrm{C}$ & SM, RS, WP, CF & $\mathrm{TH}$ \\
\hline Desmodium elegans DC. & Chamlai & $\mathrm{S}$ & $1600-1800$ & $\mathrm{C}$ & SM, RS, WP, CF & $\mathrm{SUH}, \mathrm{MH}$ \\
\hline Desmodium microphyllum (Thunb.) DC. & Sunsuni & $\mathrm{H}$ & $1600-1700$ & $\mathrm{C}$ & CF, WS, UF & $\mathrm{TH}$ \\
\hline Flemingia fruticulosa Wallich ex Benth. & Churan & $\mathrm{H}$ & $1600-2700$ & $\mathrm{C}$ & $\mathrm{CF}, \mathrm{RS}, \mathrm{WP}, \mathrm{WS}$ & WH, SUH \\
\hline Indigofera dosua Buch.-Ham. ex D.Don & Sakina & $\mathrm{S}$ & $1600-1800$ & $\mathrm{C}$ & OS, UF & $\mathrm{SUH}, \mathrm{MH}$ \\
\hline Indigofera heterantha Wall. ex Brandis & Sakina, Kathi, Kathoj & $\mathrm{S}$ & $1600-2000$ & $\mathrm{C}$ & SM, RS, WP, CF & $\mathrm{SUH}, \mathrm{MH}$ \\
\hline Trifolium repens $\mathrm{L}$. & Tipatiya & $\mathrm{H}$ & $1500-2500$ & $\mathrm{C}$ & OS, WS & $\mathrm{MH}, \mathrm{AH}$ \\
\hline $\begin{array}{l}\text { Trigonella corniculata L. } \\
\text { Fagaceae }\end{array}$ & Van methi & $\mathrm{H}$ & $2500-2800$ & $\mathrm{C}$ & UF, BO, SM & WH \\
\hline Quercus floribunda Lindl. ex Rehder & Tilonj, Moru & $\mathrm{T}$ & $2500-2800$ & $\mathrm{C}$ & SM, WC & $\mathrm{MH}$ \\
\hline Quercus leucotrichophora A. Camus & Banj & $\mathrm{T}$ & $1600-2450$ & $\mathrm{C}$ & SM, WC & $\mathrm{SUH}, \mathrm{MH}$ \\
\hline $\begin{array}{l}\text { Quercus semecarpifolia Sm. } \\
\text { Flacourtiaceae }\end{array}$ & Kharsu & $\mathrm{T}$ & $2450-2800$ & $\mathrm{C}$ & SM, WC & $\mathrm{MH}$ \\
\hline Flacourtia indica (Burm.f.) Merrill & Kandai, Kangu & S & $1600-2500$ & $\mathrm{C}$ & $\mathrm{CF}, \mathrm{RS}, \mathrm{WP}, \mathrm{WS}$ & $\mathrm{TH}$ \\
\hline Gentianaceae & & & & & & \\
\hline Crawfurdia japonica C.B. Clarke & Gimkolya & $\mathrm{H}$ & $1600-2450$ & UC & EP, WS, UF & SUH, MH \\
\hline Gentiana capitata Buch.-Ham. ex D.Don & - & $\mathrm{H}$ & $1600-2800$ & $\mathrm{C}$ & UF, OS & $\mathrm{NW}, \mathrm{MH}$ \\
\hline Gentiana pedicellata (D.Don) Wall. & Chhoti buggi & $\mathrm{H}$ & $1600-2700$ & $\mathrm{C}$ & UF, OS & SUH, MH \\
\hline Gentiana stipitata Edgew. & - & $\mathrm{H}$ & $1600-2700$ & $\mathrm{C}$ & UF, OS & $\mathrm{NWH}, \mathrm{MH}$ \\
\hline Swertia chirayita (Roxb. ex Fleming) Karsten & Chiraita & $\mathrm{H}$ & $1500-1800$ & UC & WS, UF, OS & $\mathrm{SUH}, \mathrm{MH}$ \\
\hline Swertia cordata (G.Don) C.B. Clarke & Chirata & $\mathrm{H}$ & $1800-2100$ & UC & WS, UF, OS & $\mathrm{MH}$ \\
\hline $\begin{array}{l}\text { Swertia paniculata Wall. } \\
\text { Geraniaceae }\end{array}$ & - & $\mathrm{H}$ & $1500-2000$ & UC & WS, UF, OS & $\mathrm{NWH}, \mathrm{MH}$ \\
\hline Geranium nepalense Sw. & Phori, Syunli & $\mathrm{H}$ & $1600-2700$ & C & OS, UF & $\mathrm{MH}$ \\
\hline $\begin{array}{l}\text { Geranium ocellatum Cambess } \\
\text { Hydrangeaceae }\end{array}$ & Kaphlya & $\mathrm{H}$ & $1600-2500$ & C & OS, UF, WS, RS & $\mathrm{SUH}, \mathrm{MH}$ \\
\hline Deutzia compacta Craib & Mhujvar & $\mathrm{S}$ & $1900-2800$ & $\mathrm{C}$ & WS, OS, CF & $\mathrm{MH}$ \\
\hline $\begin{array}{l}\text { Deutzia staminea R.Br. ex Wall. } \\
\text { Hypericaceae }\end{array}$ & Ghugtai & $\mathrm{S}$ & $1600-2600$ & $\mathrm{C}$ & UF, OS & WH \\
\hline Hypericum elodeoides Choisy & Basanti & $\mathrm{S}$ & $1600-3000$ & $\mathrm{C}$ & UF, OS & $\mathrm{MH}$ \\
\hline Hypericum oblongifolium Choisy & Chaya, Chitroi & $\mathrm{S}$ & $1600-1850$ & $\mathrm{C}$ & WS, UF, OS & NWH \\
\hline Hypericum uralum Buch.-Ham. ex D.Don & Bhyoul & S & $1600-2700$ & $\mathrm{C}$ & WS, RS, UF, OS & $\mathrm{TH}$ \\
\hline
\end{tabular}

Research article

CIndian Society for Education and Environment (iSee)
"Phytodiversity of Garhwal Himalaya forest" http://www.indjst.org
Sarvesh Suyal et al. Indian J.Sci.Technol. 


\begin{tabular}{|c|c|c|c|c|c|c|}
\hline Lamiaceae & (Table 1 continues) & & & & & \\
\hline Ajuga brachystemon Maxim. & Rathpathi & $\mathrm{H}$ & $1600-2500$ & C & SM, RS, WP, CF & WH \\
\hline Ajuga parviflora Benth. & Neelkanthi & $\mathrm{H}$ & $1600-2700$ & $\mathrm{C}$ & SM, RS, WP, CF & $\mathrm{TH}$ \\
\hline Clinopodium umbrosum (M.Bieb.) Koch. & Birchee & $\mathrm{H}$ & $1600-2500$ & C & OS, WS, RS, CF & $\mathrm{SUH}, \mathrm{MH}$ \\
\hline Colebrookia oppositifolia Sm. & Bindu & $\mathrm{S}$ & $1600-2400$ & C & SM, RS, WP, CF & $\mathrm{TH}$ \\
\hline Elsholtzia flava (Benth.) Benth. & - & $\mathrm{S}$ & $1600-2000$ & $\mathrm{C}$ & SM, RS, WP, CF & $\mathrm{MH}$ \\
\hline Elsholtzia fruticosa (D.Don) Rehder & Pothi & $\mathrm{s}$ & $1600-2500$ & $\mathrm{C}$ & SM, RS, WP, CF & $\mathrm{MH}$ \\
\hline Elsholtzia pilosa (Benth.) Benth. & - & $S$ & $1600-2800$ & $\mathrm{C}$ & SM, RS, WP, CF & $\mathrm{MH}$ \\
\hline Lamium album $\mathrm{L}$. & Tilka & $\mathrm{H}$ & $1700-2800$ & $\mathrm{C}$ & SM, RS, WP, CF & $\mathrm{SUH}, \mathrm{MH}$ \\
\hline Leucas lanata Benth. & Bis-kapra, Gumma & $\mathrm{H}$ & $1600-2300$ & C & WS, CF, OS & WH \\
\hline Mentha arvensis $\mathrm{L}$. & Paudina & $\mathrm{H}$ & $1600-2200$ & $\mathrm{C}$ & WS, WC, BO,SM & WH \\
\hline Mentha sylvestris L. & Paudina & $\mathrm{H}$ & $1600-2500$ & $\mathrm{C}$ & $\mathrm{MH}$ & WH \\
\hline Micromeria biflora (Buch.-Ham. ex D.Don) & Ban-ajwain & $\mathrm{H}$ & $1600-2100$ & $\mathrm{C}$ & OS, SM & SUH, MH \\
\hline Benth. & & & & & & \\
\hline Origanum vulgare L. & Bantulsi & $\mathrm{H}$ & $1600-2300$ & $\mathrm{C}$ & SM, RS, WP, CF & $\mathrm{MH}$ \\
\hline Phylomis bracteosa Royle ex Benth & - & $\mathrm{H}$ & $2500-2800$ & $\mathrm{C}$ & SM, RS, WP, CF & SUH, MH \\
\hline Phylomis macrophylla Wall.ex Benth & - & $\mathrm{H}$ & $2500-2800$ & C & SM, RS, WP, CF & $\mathrm{SUH}, \mathrm{MH}$ \\
\hline Plectranthus mollis (Aiton) Sperngel & - & $\mathrm{H}$ & $1600-2500$ & $\mathrm{C}$ & WS, SM, BO, CF & $\mathrm{WH}$ \\
\hline Prunella vulgaris $\mathrm{L}$. & - & $\mathrm{H}$ & $1600-2700$ & $\mathrm{C}$ & SM, RS, WP, CF & $\mathrm{MH}$ \\
\hline Rabdosia rugosa (Wall. Ex Benth.) Hara & Chichri & $S$ & $1650-2600$ & $\mathrm{C}$ & SM, CF,WS & WH \\
\hline Salvia lanata Roxb. & Ghanyajhar, Ghaniya & $\mathrm{H}$ & $1600-2500$ & C & WS, OS, CF & MH \\
\hline Salvia nubicola Wall. Ex Sweet & Ganya & $\mathrm{H}$ & $1600-2200$ & C & WS, OS, CF & $\mathrm{MH}$ \\
\hline Scutellaria repens Buch.-Ham & - & & $1800-2550$ & $\mathrm{C}$ & WS, OS, CF, UF & $\mathrm{SUH}, \mathrm{MH}$ \\
\hline Scutellaria scandens Buch.-Ham. ex D.Don & Kutlaphul & $\mathrm{H}$ & $1600-2200$ & C & SM, RS, WP, CF & $\mathrm{SUH}, \mathrm{MH}$ \\
\hline Thymus linearis Benth. & Ban ajwain & $\mathrm{H}$ & $2000-3000$ & C & OS, UF, SM & \\
\hline $\begin{array}{l}\text { Lauraceae } \\
\text { Neolitsea pallens (D.Don) Memiyama \& Hara }\end{array}$ & & & & & & \\
\hline $\begin{array}{l}\text { Neolitsea pallens (D.Don) Memiyama \& Hara } \\
\text { ex Hara }\end{array}$ & Bilaru & $\mathrm{T}$ & $1600-2200$ & C & SM, UF & $\mathrm{SUH}, \mathrm{MH}$ \\
\hline $\begin{array}{l}\text { Persea duthiei (King ex Hook. F.) Kostermans } \\
\text { Liliaceae }\end{array}$ & Kaula Sairi, Bhadrao & $\mathrm{T}$ & $1600-2200$ & C & SM, UF & $\mathrm{SUH}, \mathrm{MH}$ \\
\hline Asparagus adscendens Buch.-Ham. ex Roxb. & Sharanoi, Kaunta & $\mathrm{H}$ & $1600-2000$ & UC & WS, RS, DS, OS, CF & SUH, MH \\
\hline $\begin{array}{l}\text { Reinwardtia indica Dum. } \\
\text { Lythraceae }\end{array}$ & Phiyunli & $\mathrm{S}$ & $1600-1900$ & C & SM, RS, WP, CF & $\mathrm{SUH}, \mathrm{MH}$ \\
\hline Woodfordia fruticosa (L.) Kurz & Dhaula & $\mathrm{S}$ & $1700-1550$ & C & SM, RS, WP, CF & SUH \\
\hline Malvaceae & & & & & & \\
\hline $\begin{array}{l}\text { Sida acuta Burm. F. } \\
\text { Moraceae }\end{array}$ & Karenti, Bariara & $\mathrm{H}$ & $1650-2000$ & C & WS, OS, CF, UF & $\mathrm{OH}, \mathrm{SHB}$ \\
\hline $\begin{array}{l}\text { Ficus hederacea Roxb. } \\
\text { Myricaceae }\end{array}$ & Beduli, Laduli & $\mathrm{Cl}$ & $1600-2500$ & $\mathrm{C}$ & UF, SM & $\mathrm{SUH}, \mathrm{MH}$ \\
\hline $\begin{array}{l}\text { Myrica esculenta Buch.-Ham. ex D.Don } \\
\text { Myrsinaceae }\end{array}$ & Kaphal & $\mathrm{T}$ & $1600-2200$ & C & SM, UF, WS, BO & $\mathrm{MH}$ \\
\hline Myrisine semiserrata Wall. & Gaunta, Bains & $\mathrm{S}$ & $1600-2500$ & $\mathrm{C}$ & WS, CF, OS & $\mathrm{SUH}, \mathrm{MH}$ \\
\hline $\begin{array}{l}\text { Myrisine africana } \mathrm{L} . \\
\text { Oleaceae }\end{array}$ & Chupra, Paharicha & $\mathrm{S}$ & $1600-2100$ & $\mathrm{C}$ & UF, SM & $\mathrm{SUH}, \mathrm{MH}$ \\
\hline Jasminum humile $\mathrm{L}$. & Surmarchi, Pilichameli & $\mathrm{S}$ & $1600-2800$ & C & BO, WS, SM WC, UF & WH \\
\hline Jasminum officinale $\mathrm{L}$. & Jai, Champa & $\mathrm{Cl}$ & $1600-2300$ & $\mathrm{C}$ & WS, BO & $\mathrm{WH}$ \\
\hline Orchidaceae & & & & & & \\
\hline Calanthe plantaginea Lindl. & - $\quad$ & $\mathrm{H}$ & $1600-2200$ & $\mathrm{C}$ & SM, RS, WP, CF & $\mathrm{MH}$ \\
\hline Goodyera fusca (Lindl.) Hook.f. & Guddara & $\mathrm{H}$ & $2500-3000$ & $\mathrm{C}$ & CF, RS, WP, WS & $\mathrm{MH}, \mathrm{AH}$ \\
\hline Goodyera repens (L.) R.Br. & Girwara & $\mathrm{H}$ & $1600-1900$ & $\mathrm{C}$ & SM & $\mathrm{MH}, \mathrm{AH}$ \\
\hline $\begin{array}{l}\text { Herminium lanceum (Thunb. ex Sw.) Vujik } \\
\text { Oxalidaceae }\end{array}$ & Jalya & $\mathrm{H}$ & $1600-2500$ & $\mathrm{C}$ & SM, RS, WP, CF & $\mathrm{MH}, \mathrm{AH}$ \\
\hline Oxalis corniculata $\mathrm{L}$. & Bhilmori & $\mathrm{H}$ & $1600-2700$ & $\mathrm{C}$ & SM, RS, WP, CF & $\mathrm{TH}$ \\
\hline Oxalis dehradunensis Raizada & Khatura & $\mathrm{H}$ & $1600-2300$ & $\mathrm{C}$ & SM, RS, WP, CF & $\mathrm{OH}, \mathrm{SUH}$ \\
\hline Pinaceae & & & & & & \\
\hline Abies pindrow Royle & Raga & $\mathrm{T}$ & $2400-2800$ & C & BO, SM, OS & $\mathrm{MH}, \mathrm{WH}$ \\
\hline Abies spectabilis (D.Don) & Morinda, Raga & $\mathrm{T}$ & $2500-2700$ & UC & $\mathrm{BO}, \mathrm{SM}, \mathrm{OS}$ & \\
\hline Pinus roxburghii Sarg. & Chir, Kulain & $\mathrm{T}$ & $1600-1900$ & $\mathrm{C}$ & $\mathrm{BO}, \mathrm{DS}$ & $\mathrm{SUH}, \mathrm{MH}$ \\
\hline Plantaginaceae & & & & & & \\
\hline Plantago depressa Willd. & Luhurya & $\mathrm{H}$ & $1600-2100$ & C & SM, RS, WP, CF & WH \\
\hline $\begin{array}{l}\text { Plantago erosa Wall. } \\
\text { Poaceae }\end{array}$ & Luhurya, Lahyrya & $\mathrm{H}$ & $1600-22000$ & $\mathrm{C}$ & SM, RS, WP, CF & $\mathrm{MH}, \mathrm{AH}$ \\
\hline Synarundinaria anceps (Mitf.) Chao \& Revoize & Saruru, Ringal & $\mathrm{S}$ & $2400-2650$ & C & SM, WP, CF & WH \\
\hline Cynodon dactylon (L.) Persoon & Dubla & $\mathrm{G}$ & $1600-1850$ & $\mathrm{C}$ & SM, RS, WP, CF & $\mathrm{TH}$ \\
\hline Paspalum paspalodes (Mich.) Scribn. & - & & $1850-2250$ & C & WS, CF, OS & $\mathrm{WH}, \mathrm{MH}$ \\
\hline Sinarundinaria falcate (Nees) Chao \& Renoize & Gad-Ringal, Ringal & G & $1500-2000$ & $\mathrm{C}$ & SM, RS, WP, UF & $\mathrm{WH}, \mathrm{MH}$ \\
\hline Thamnocalamus falconeriHook.f. ex Munro & Dev-Ringal & G & $1500-2000$ & C & SM, RS, WP, UF & $\mathrm{MH}$ \\
\hline Thamnocalamus spathiflora (Trinius) Munro & Tham Ringal & G & $2500-2000$ & $\mathrm{C}$ & SM, RS, WP, UF & $\mathrm{WH}, \mathrm{MH}$ \\
\hline
\end{tabular}

Research article

CIndian Society for Education and Environment (iSee)
"Phytodiversity of Garhwal Himalaya forest" http://www.indjst.org
Sarvesh Suyal et al. Indian J.Sci.Technol. 
Polygonaceae (Table 1 continues....)

Persicaria capitata (Buch.-Ham. ex D.Don)

H.Gross

Polygonatum cirrhifolium (Wall.) Royle

Polygonatum verticillatum (L.) Allioni

Polygonum capitatum Buch.-Ham. ex D.Don

Polygonum donii Meissn.

Polygonum pterocarpum Wall. ex Meissn.

Rumex hastatus D.Don

Rumex nepalensis Spreng.

Primulaceae

Androsace rotundifolia Hardwicke

Primula denticulata Sm.

Ranunculaceae

Anemone obtusiloba D.Don

Anemone rivularis Buch.-Ham. ex DC.

Anemone vitifolia Buch.-Ham. ex DC.

Artemisia roxburghiana Wallich ex Besser

Clematis barbellata Edgew.

Clematis buchananiana DC.

Clematis connata DC.

Clematis montana Buch.-Ham. ex DC.

Delphinium denudatum Wall. Ex Hook.f.

Ranunculus laetus Wall. Ex D.Don

Thalictrum cultratum Wall.

Thalictrum foliolosum DC.

Rhamnaceae

Rhamnus virgatus Roxb.

Rosaceae

Agrimonia pilosa Ledebour

Cotoneaster acuminatus Lind.

Cotoneaster bacillaris Wallich

Cotoneaster microphyllus Wall. Ex Lindl

Duchesnea indica (Andr) Focke

Fragaria nubicola Lindl ex Lacaita

Potentilla fulgens Wall. Ex Hook.

Prinsepia utilis Royle

Pyracantha crenulata(D.Don) M. Roemer

Pyrus pashia Buch. Ham. ex D.Don

Rosa brunonii Lindl.

Rosa macrophylla Lindl.

Rosa sericea Lindl.

Rubus biflorus Buch.-Ham.ex Smith

Rubus ellipticus Sm.

Rubus foliolosus D.Don

Rubus nepalensis (Hook.f.) Kuntze

Rubus niveus Thunb.

Rubus paniculatus Sm.

Spiraea canescens D.Don

Rubiaceae

Galium aparine L.

Galium asperifolium Wall.

Galium elegans Wall.

Leptodermis lanceolata Wall.

Himrandia tetrasperma (Roxb.) Yamazaki

Rubia manjith Roxb. ex Fleming

Spermadictyon sauveolens Roxb.

Rutaceae

Boenninghausenia albiflora (Hook.) Reichb.

ex Meisn

Murrraya paniculata (L.) Jack

Skimmia anquetilia Taylor \& Airy Shaw

Zanthoxylum armatum DC.

Saxifragaceae

Bergenia ciliata (Haworth) Sternberg

Scrophulariaceae

Hemiphragma heterophyllum Wall.

Verbascum thapsus $\mathrm{L}$.

Kaflya

Khakan, Medha Kantula, Mahameda -

-

Kilmori, Almoru

Khatur

Jalkutra

Kanchphool, Kakrya

Mirchilee, Angeli

Mudeela

Kunjaa, Chamur

Kanrya, Santai

Lagulia

Kujju

-

$-$

Mamiri

Choudelu

Lesukuri

Cham-ruins

Ruins, Rensu

Bugarchilla

Bhiun-kaphal

Gand-Kaphal

Bajradanti

Bhenkuli

Ghingaru

Melu

Kujju

Ban-gulab

Dhurkunja

Hinsara, Achanoi

Hinssar,Hisalu,Hinshoi

Kala Hissar

Gangoor

Anchu

Kall-Hinsar

Jhar Mairala

Kuri

Leswakuri

Kutub, Manjeethee

Padera, Padar

Kamoli, Ghara

Manjeet, Lichkuru

Padera

Pishumar

Machula, Kamini

Nairpatti, Patrang

Timroo

Silpara

Akulbir, Kakri

Tamakhu
H $\quad 1600-2300$ C WS, SM, BO

$\begin{array}{llll}1600-2300 & \text { UC } & \text { SM, CF,WS } \\ \text { H } & 1600-2650 & \text { UC } & \text { SM, CF,WS }\end{array}$

H 1600-1900 C WS, SM, BO, CF

H $1600-2100$ C WS, SM, BO, CF

H 1650-1900 C SM, CF,WS

H 1600-2100 C RS, WP, CF, DS

H $1600-2300$ C WP, RS, CF, WP

C BO, RS, WC, WS

H $1600-2200$

H $1800-2250$

C SM, WS, MP, BO, WC

H 2500-2650 C OP, WS, UF, SM

H 1600-2500 C WS, SM, CF

H 1850-2250 C WS, SM, CF

H $1650-2250$ C OP, WS, UF, SM

Cl 1600-2000 C UF

Cl 1600-3000 UC UF

Cl 1600-3000 C UF, OS, WS, WC

Cl 1600-2500 C UF, WS

H $1600-2400$ C WS, OS, CF

H 1600-2600 C SM, OS, WS, CF

H 1500-2500 C WS, SM, CF

H 1500-2500

C WS, SM, CF

S $1600-2200$

C WS, OS

\section{0-2800}

2450-2700

2000-2800

$1600-2500$

$1600-2000$

1600-2000

2550-2800

$1600-2100$

1600-2300

$1600-2200$

Cl 1600-2300

$1600-2800$

$2200-2700$

2200-2600

$1600-2300$

$1600-2350$

$1600-2300$

1600-2200

$1600-2780$

1600-3000

C UF, OS, SM

C UF, OS, WS, WC

C UF, OS, WS, WC

C BO, OS

C UF, WS

C WS, SM, UF, OS

C OS, UF, SM

C WS, OS, CF

C OS, WS

C UF, WS, SM, CF

C OS, WS, RS

UC SM, CF,WS, RS

C OS

C OS, WS, RS

C OS, DS, WS, RS, CF

C OS, SM, WS, RS, CF

C SM,WS

C OS, WS

C OS,WS

C WS, RS, OS

$1600-2500$

$1600-2500$

C WS, UF, SM

C WS, UF, SM

C CF, RS, WP, WS

$1600-2000$

$1600-2300$

1600-2300

$1500-1700$

C WS, CF, OP

C UF, WS, RS

C UF, WS, RS, CF

C RS, WS, OP

H $\quad 1600-2700$

C UF, SM, WC

S 1600-2200 C RS

S 1500-3000

S 1650-1800

C WS, OS, CF, UF

H $1600-2500$

C SM, UF, BO

H $1800-2800$ C SM, OS, UF

H $1800-2500$

C OP, WP, WS, SM, WC, CF
SUH, MH

$\mathrm{MH}$

$\mathrm{MH}, \mathrm{AH}$

$\mathrm{WH}, \mathrm{MH}$

$\mathrm{WH}, \mathrm{MH}$

$\mathrm{WH}, \mathrm{MH}$

$\mathrm{SUH}, \mathrm{MH}$

$\mathrm{MH}, \mathrm{AH}$

$\mathrm{MH}$

$\mathrm{MH}$

$\mathrm{AH}, \mathrm{MH}$

$\mathrm{AH}, \mathrm{MH}$

$\mathrm{AH}, \mathrm{MH}$

WH

NWH

$\mathrm{MH}, \mathrm{SUH}$

$\mathrm{MH}$

$\mathrm{MH}, \mathrm{SUH}$

$\mathrm{MH}, \mathrm{SUH}$

$\mathrm{TH}$

$\mathrm{TH}$

$\mathrm{TH}$

SUH, MH

$\mathrm{MH}$

$\mathrm{MH}$

$\mathrm{MH}$

$\mathrm{MH}$

$\mathrm{TH}$

$\mathrm{MH}$

$\mathrm{MH}$

$\mathrm{MH}$

SUH

$\mathrm{SHB}, \mathrm{MH}$

$\mathrm{MH}, \mathrm{SUH}$

$\mathrm{MH}$

$\mathrm{MH}$

$\mathrm{MH}, \mathrm{SUH}$

$\mathrm{SUH}, \mathrm{MH}$

$\mathrm{MH}$

$\mathrm{MH}$

$\mathrm{MH}$

$\mathrm{MH}$

$\mathrm{MH}$

$\mathrm{MH}, \mathrm{AH}$

$\mathrm{MH}, \mathrm{AH}$

$\mathrm{MH}, \mathrm{AH}$

$\mathrm{MH}$

SUH

$\mathrm{TH}$

SUH, MH

SUH, MH

SHB

$\mathrm{MH}$

$\mathrm{SHB}$

NWH

$\mathrm{MH}$

$\mathrm{SUH}, \mathrm{MH}$

Simaroubaceae

Picrasma quassioides (D.Don) Bennett

Karui, Kakra

S $\quad 1600-24000$ UC BO, DS

$\mathrm{OH}$

Research article

CIndian Society for Education and Environment (iSee)
"Phytodiversity of Garhwal Himalaya forest" http://www.indjst.org

Sarvesh Suyal et al. 


\begin{tabular}{|c|c|c|c|c|c|c|}
\hline Smilacaceae & (Table 1 continues....) & & & & & \\
\hline Smilax aspera L. & Kukardara & $\mathrm{Cl}$ & $1500-2000$ & $\mathrm{C}$ & OS, WS, UF & $\mathrm{MH}, \mathrm{SUH}$ \\
\hline \multicolumn{7}{|l|}{ Solanaceae } \\
\hline Solanum erianthum D.Don & Akra & $\mathrm{S}$ & $1500-2000$ & $\mathrm{C}$ & RS, WS & $\mathrm{SHB}$ \\
\hline Solanum nigrum L. & Makoi, Kirmoi & $\mathrm{H}$ & $1500-2000$ & $\mathrm{C}$ & RS, WS & $\mathrm{TH}$ \\
\hline \multicolumn{7}{|l|}{ Symplocaceae } \\
\hline $\begin{array}{l}\text { Symplocos paniculata (Thunb.) Miq. } \\
\text { Taxaceae }\end{array}$ & Lodh & $\mathrm{T}$ & $2000-2500$ & $\mathrm{C}$ & UF, SM & $\mathrm{MH}, \mathrm{SUH}$ \\
\hline $\begin{array}{l}\text { Taxus baccata L. } \\
\text { Thymelaeaceae }\end{array}$ & Thuner & $\mathrm{T}$ & $2200-2700$ & UC & UF, BO, SM & $\mathrm{MH}$ \\
\hline $\begin{array}{l}\text { Daphne papyracea Wall. ex Steudel } \\
\text { Ulmaceae }\end{array}$ & Satpura & $\mathrm{S}$ & $1600-2700$ & UC & UF, SM, WC & $\mathrm{MH}$ \\
\hline $\begin{array}{l}\text { Ulmus wallichiana Planchon } \\
\text { Urticaceae }\end{array}$ & \multicolumn{5}{|c|}{ Urticaceae } & $\mathrm{WH}, \mathrm{MH}$ \\
\hline Debregeasia salicifolia (D.Don) Rendle & Syanru & S & $1600-2000$ & $\mathrm{C}$ & WS, CF,SM & $\mathrm{SUH}, \mathrm{MH}$ \\
\hline Girardinia diversifolia (Link) Friis & Bhainsya Kandali & $\mathrm{H}$ & $1600-2200$ & $\mathrm{C}$ & RS, WS, CF & $\mathrm{SUH}, \mathrm{MH}$ \\
\hline Urtica ardens Link & - & $\mathrm{S}$ & $1600-2500$ & $\mathrm{C}$ & $\begin{array}{l}\text { OP, WP, WS, RS, SM, WC, } \\
\text { CF }\end{array}$ & $\mathrm{SUH}, \mathrm{MH}$ \\
\hline Urtica dioica $\mathrm{L}$. & Kandali & $\mathrm{S}$ & $1600-2500$ & $\mathrm{C}$ & $\begin{array}{l}\text { OP, WP, WS, RS, SM, WC, } \\
\text { CF }\end{array}$ & $\mathrm{MH}, \mathrm{SUH}, \mathrm{NWH}$ \\
\hline \multicolumn{7}{|l|}{ Valerianaceae } \\
\hline Valeriana hardwickii Wall. & Shammia & $\mathrm{H}$ & $1600-2200$ & $\mathrm{C}$ & SM, CF & $\mathrm{MH}$ \\
\hline Valeriana jatamansii Jones & Balchhari, Sumaya & $\mathrm{H}$ & $2200-2700$ & $\mathrm{C}$ & UF, BO, SM & $\mathrm{MH}$ \\
\hline \multicolumn{7}{|l|}{ Verbenaceae } \\
\hline Callicarpa macrophylla Vahl & Daiya, Bhirmoli & $\mathrm{H}$ & $1600-1800$ & $\mathrm{C}$ & WC, SM, WS & $\mathrm{SUH}, \mathrm{MH}$ \\
\hline Lantana camara $\mathrm{L}$. & Kuri-ghas, Laltenya & S & $1600-2300$ & $\mathrm{C}$ & SM, RS, WP, CF & $\mathrm{TH}$ \\
\hline \multicolumn{7}{|l|}{ Violaceae } \\
\hline Viola betonicifolia Sm. & Vanfsa, Phori & $\mathrm{H}$ & $1800-2500$ & $\mathrm{C}$ & UF, SM & $\mathrm{SUH}$ \\
\hline Viola biflora L. & Vanfsa & $\mathrm{H}$ & $2200-2700$ & $\mathrm{C}$ & UF, SM & $\mathrm{AH}, \mathrm{MH}$ \\
\hline Viola canescens Wall. & Vanfsa & $\mathrm{H}$ & $1600-2000$ & $\mathrm{C}$ & WS, SM, CF & $\mathrm{MH}$ \\
\hline Viola pilosa Blume & Vanfsa & $\mathrm{H}$ & $1600-2000$ & $\mathrm{C}$ & WS, SM, CF & $\mathrm{MH}, \mathrm{AH}$ \\
\hline Viola serpens Wall. Ex Gingins & Kauru & $\mathrm{H}$ & $1600-2200$ & $\mathrm{C}$ & $\begin{array}{l}\text { OP, WP, WS, RS, SM, WC, } \\
\text { CF }\end{array}$ & $\mathrm{MH}, \mathrm{AH}$ \\
\hline \multicolumn{7}{|l|}{ Vitaceae } \\
\hline Parthenocissus semicordata (Wall.) Planchon & Laguli & $\mathrm{Cl}$ & $1600-2800$ & C & $\mathrm{BO}, \mathrm{WS}$ & $\mathrm{SUH}, \mathrm{MH}, \mathrm{NWH}$ \\
\hline \multicolumn{6}{|l|}{ Zingiberaceae } & $\mathrm{MH}$ \\
\hline Hedychium spicatum Buch.-Ham. & Ban-Haldi & $\mathrm{H}$ & $1600-2500$ & $\mathrm{C}$ & BO, WS, SM WC, UF & SUH, MH \\
\hline Roscoea purpurea Smith & Kakoli & $\mathrm{H}$ & $1850-2700$ & $\mathrm{C}$ & OS, WS, RS & $\mathrm{MH}$ \\
\hline \multicolumn{7}{|c|}{$\begin{array}{c}\text { BO= Bouldry; C= Common; CF= Cultivated Fields; Cl= Climber; DS= Dry Slopes; EP= Epiphytes; G= Grass; H= Herb; LF= Life Form; Oc= } \\
\text { Occurrence; OS= Open Slopes; PA= Parasite; RS= Roadside; S= Shrub; SM=Shady Moist; T= Tree; UC= Uncommon; UF= Under Forest; } \\
\text { WC= Water Courses; WP= Waste Places; WS= Wayside. TH= Throughout Himalaya; } \text { AH= Alpine Himalaya, SUH= Subalpine Himalaya; SAH= } \\
\text { Subalpine Himalaya; NWH= North-west Himalaya; MH= Montane Himalaya; WH= West Himalaya; OH= Outer Himalaya; SHB= Sub Himalayan } \\
\text { belt; CH= Central Himalaya; }\end{array}$} \\
\hline
\end{tabular}

Table 2. Comparison of floristic diversity of the Chaurangikhal forest area with other floras

\begin{tabular}{|l|l|c|c|c|c|c|}
\hline \multicolumn{1}{|c|}{ Flora } & \multicolumn{1}{c|}{ Source } & $\mathrm{F}$ & $\mathrm{G}$ & $\mathrm{S}$ & Ratio (F:G) & Ratio (G:S) \\
\hline Chaurangikhal, Garhwal, UK. & Present Study & 69 & 159 & 231 & $1: 2.30$ & $1: 1.45$ \\
\hline Mandal-Chopta, Garhwal, UK. & Gairola et al., 2009b & 93 & 249 & 338 & $1: 2.68$ & $1: 1.36$ \\
\hline Chamoli Garhwal, UK & Naithani, 1984-1985 & 163 & 892 & 1934 & $1: 5.47$ & $1: 2.17$ \\
\hline Garhwal Himalaya, UK. & Gaur, 1999 & 189 & 978 & 2035 & $1: 5.17$ & $1: 2.08$ \\
\hline British India. & Hooker, 1872-1897 & 174 & 2346 & 14384 & $1: 13.48$ & $1: 6.13$ \\
\hline Mussorie, UK. & Raizada \& Saxena, 1978 & 131 & 649 & 1219 & $1: 4.95$ & $1: 1.88$ \\
\hline Himachal Pradesh. & Chowdhery \& Wadhwa, 1984 & 180 & 1093 & 3200 & $1: 6.07$ & $1: 2.93$ \\
\hline Bashahr, HP. & Nair, 1977 & 134 & 689 & 1579 & $1: 5.14$ & $1: 2.29$ \\
\hline Shimla, HP. & Collett, 1902 & 123 & 639 & 1326 & $1: 5.20$ & $1: 2.08$ \\
\hline Lahual-Spiti, HP. & Aswal \& Mehrotra, 1994 & 79 & 353 & 985 & $1: 4.47$ & $1: 2.80$ \\
\hline Kullu, HP. & Sharma \& Dhaliwal, 1997 & 126 & 504 & 930 & $1: 4.00$ & $1: 1.85$ \\
\hline Sirmour, HP. & Kaur \& Sharma, 2004 & 139 & 544 & 898 & $1: 3.92$ & $1: 1.65$ \\
\hline
\end{tabular}

$U K=$ Uttarakhand; $H P=$ Himachal Pradesh; $F=$ Family; $G=$ Genus; $S=$ Species

Research article

CIndian Society for Education and Environment (iSee)
"Phytodiversity of Garhwal Himalaya forest" http://www.indjst.org
Sarvesh Suyal et al. Indian J.Sci.Technol. 
important medicinal plants. The specimens of each species were collected and identified with the help of floras (Naithani, 1984-85; Gaur, 1999) and existing herbaria of Botany department HNB Garhwal University (GUH), forest research institute (DD) and botanical survey of India, northern circle (BSD). After identification, the plants were arranged according to Bentham and Hooker's system of classification (1862-1883). Taxonomical categories-genera and species within the family were placed alphabetically and species were described with usual citations. Information about vernacular names, habitat, life form, altitudinal range and occurrence were collected for each species. The twelve types of habitats were identified in the study area viz., bouldery, cultivated fields, dry slopes, epiphytes, open slopes, parasite, roadside, shady moist, under forest, water courses, waste places and wayside. Occurrence was categorized into common and uncommon types.

Results

The results of the study are placed in the Table 1 . A total of 231 species belonging to 159 genera and 69 families were recorded. Of these, gymnosperms were represented by 4 species, 3 genera and 2 families, dicotyledons by 212 species, 143 genera and 62 families and monocotyledons by 15 species, 13 genera and 5 families (Fig. 3). In the study area, the ratio of family to genera was $1: 2.30$, family to species was $1: 3.35$ and genera to species was 1: 1.45 . Among all the species recorded the $88.31 \%$ (204 spp.) of the total species had common occurrence, whereas rest $11.69 \%$ (27 spp.) of the species had uncommon occurrence in the study area.

In gymnosperms, Pinaceae ( 3 species \& 2 genera) was the dominant family. Among the families of angiosperms Lamiaceae was the dominant family with 23 species and 16 genera followed by Asteraceae ( 21 species \& 13 genera), Rosaceae (20 species \& 11 genera), Ranunculaceae (12 species \& 6 genera), Fabaceae (8 species \& 6 genera), Caryophyllaceae (8 species \& 5 genera), Polygonaceae (8 species \& 3 genera), Rubiaceae ( 7 species \& 5 genera), Gentianaceae (7 species \& 3 genera), Poaceae (6 species \& 5 genera), Violaceae ( 5 species \& 1 genera), Rutaceae (4 species \& 4 genera), Orchidaceae (4 species \& 3 genera), Caprifoliaceae (4 species \& 2 genera), Ericaceae (3 species \& 3 genera), Euphorbiaceae ( 3 species \& 2 genera), Berberidaceae ( 3 species \& 1 genera), Scrophulariaceae (5 species \& 4 genera), Solanaceae (5 species \& 3 genera), Asclepiadaceae ( 4 species \& 4 genera), Brassicaceae ( 4 species \& 4 genera), Ericaceae ( 4 species \& 3 genera), Lauraceae ( 4 species $\& 3$ genera), Moraceae ( 4 species \& 1 genera) and Violaceae (4 species \& 1 genera). Dominant genera of the study area was Rubus with 6 species followed by Polygonum \& Viola (5 spp. each), Clematis \& Senecio (4 spp. each), Anemone, Berberis, Cotoeaster, Elsholtzia, Galium, Gentiana, Hypericum, Quercus, Rosa, Swertia \& Viburnum (3 spp. each).
Genera whose two species were recorded in the study area were Ainsliaea, Ajuga, Anaphalis, Cerastium, Cirsium, Desmodium, Deutzia, Dioscorea, Erigeron, Geranium, Goodyera, Indigofera, Inula, Jasminum, Mentha, Myrisine, Oxalis, Phylomis, Plantago, Rumex, Salvia, Scitellaria, Silene, Solanum, Stellaria, Thalictrum, Thamnocalamus, Urtica and Valeriana.

\section{Discussion}

Tropical Montane Himalayan forests are characterized by strong gradients related to topography and manifest as differences in elevation, precipitation, humidity, soil type, slope, aspect, and radiation. Species adapt to these gradients in often contrasting ways, and their distribution depends on the characteristics that define their reproduction and survival (Young et al., 2002). Trees, the most important functional group in the ecosystem, were most diverse at middle elevations, but species composition changes over multiple gradients. Forest communities are subject to periodic disturbances from landslides due to high precipitation and mountainous terrain (Veblen et al., 1981). Because of disturbances and multiple gradients, montane Himalayan forests are extraordinarily complex spatially; thus, habitat diversity and species turnover are prominent attributes of this ecosystem. Lower montane Himalayan forest communities are similar to lowland forests; however, with increasing altitude, montane Himalayan species become more abundant and lowland species more rare.

Inventory and monitoring of biodiversity of any area is prerequisite for conservation and management planning. Hooker (1906) described Orchidaceae, Fabaceae, Poaceae, Rubiaceae, Euphorbiaceae, Acanthaceae, Asteraceae, Cyperaceae, Lamiaceae and Urticaceae as 10 dominant families of India, whereas recently Gairola et al. (2009b) recorded Asteraceae, Lamiaceae, Rosaceae, Orchidaceae, Poaceae, Urticaceae, Polygonaceae, Fabaceae, Ranunculaceae and Euphorbiaceae as 10 dominant families in Mandal-Chopta forest of Garhwal Himalaya. In Chaurangikhal forest area 10 dominant families were recorded as Lamiaceae, Asteraceae, Rosaceae, Ranunculaceae, Fabaceae, Caryophyllaceae, Polygonaceae, Rubiaceae, Gentianaceae and Poaceae. The comparative account of the ratio of the families with genera and genera with species of various floras with the Chaurangikhal forest area has been presented in Table 2 . From the table, it is clear that these ratios are directly proportional to the biogeographic area. It is believed that out of over 1600 species of medicinal plants traditionally used in India (Uniyal et al., 2002), more than $50 \%$ species come from the Himalayan region. About 2,500 wild plant species are reported in use for medicinal purposes in Indian sub-continent, of which, possibly about 300 taxa are used in 8,000 licensed pharmaceuticals in India (Ahmad, 1993). On the basis of our data, it is possible to affirm that there is high diversity of medicinal plants in the Chaurangikhal forest area. On the other hand, the importance of conservation of these medicinal plants is
Research article

CIndian Society for Education and Environment (iSee)
"Phytodiversity of Garhwal Himalaya forest" http://www.indjst.org
Sarvesh Suyal et al. Indian J.Sci.Technol. 
unquestionable, because this knowledge represents additional data for selecting plants that also should be used in studies focusing on ecosystem conservation.

Chaurangikhal forest was found to be very rich in phytodiversity. Unplanned use and habitat degradation may lead to depletion of the species from the area. Further, habitat wise monitoring of the species using standard ecological methods is suggested. The present study provides comprehensive information on species diversity, altitudinal and habitat wise distribution pattern of higher plants of the Chaurangikhal forest area. This study will be of great help to the state govt., particularly the forest dept. in developing a strategy and action plan for the management of this biodiversity rich forest area.

\section{Acknowledgements}

We thankfully acknowledge the financial support provided by the Department of Science and Technology, Government of India, New Delhi, vide its Project No. SP/SO/PS-52/2004 and Uttarakhand forest Dept. for providing meteorological data.

\section{References}

1. Ahmad RU (1993) Medicinal plants used in ISM-their procurement, cultivation, regeneration and import/export aspects- a report. In: Govil JN, Singh VK, Shamima H, eds., Glimpses in Plant Res. Vol X. Medicinal Plants: New Vistas of Res. Part-I. Today's \& Tomorrow's Printers and Publishers, Delhi. pp:221-258.

2. Aswal BS and Mehrotra BN (1994) Flora of Lahaul-Spiti. Bishen Singh Mahendra Pal Singh, Dehradun. pp:172.

3. Bentham G and Hooker JD (1883) Genera Plantarum. London, 3 Vols.

4. Chowdhery HJ and Wadhwa BM (1984) Flora of Himachal Pradesh, Vol. 1-3. Botanical Survey of India, Calcutta.

5. Collett $H$ (1902) Flora simlensis: A handbook of flowering plants of Simla and neighborhood. Thacker Spink \& Co., London.

6. Farooquee NA and Saxena KG (1996) Conservation and utilization of medicinal plants in high hills of the central Himalayas. Environ. Conserv. 23, 75-80.

7. Forest survey of India (FSI) (2003) The state of forest report, Dehradun, FSI, Ministry of environment and forests, Govt of India, New Delhi. pp:134.

8. Gairola S, Sharma CM, Ghildiyal SK, Suyal S, Rana CS and Butola DS (2009b) Biodiversity conservation and sustainable rural development in the Garhwal Himalaya. Report Opin. 1(4), 6-12.

9. Gairola S, Sharma CM, Rana CS, Ghildiyal SK and Suyal S (2009a) Phytodiversity (angiosperms and gymnosperms) in Mandal-Chopta forest of Garhwal Himalaya, Uttarakhand, India. Nature Sci. 8(1), 1-17.

10. Gaur RD (1999) Flora of the district Garhwal northwest Himalaya (with ethanobotanical notes). Transmedia Publication, Srinagar (Garhwal) India.

11. Hooker JD (1872-1897) Flora of British India. Vol. 1-7, Reeve and Company, London.

12. Hooker JD (1906) A sketch of flora of British India. London.

13. Kaur H and Sharma M (2004) Flora of Sirmaur (Himachal Pradesh). Bishen Singh Mahendra Pal Singh, Dehradun.
Vol. 3 No. 3 (Mar 2010)

ISSN: 0974- 6846

14. Mathur VB, Kathyat JS and Rath DP (2000) Envis Bulletin: Wildlife and Protected Areas Vol. 3(1). Wildlife Institute of India, Dehradun.

15. Nair NC (1977) Flora of Bashahar Himalaya. Today and tomorrow publications, Delhi.

16. Naithani BD (1984-1985) Flora of Chamoli. 2 Vols. BSI, Howrah.

17. Negi KS and Gaur RD (1994) Principal wild food plants of W. Himalaya, Uttar Pradesh, India. In: Higher plants of Indian subcontinent. 3, 1-147.

18. Raizada MB and Saxena HO (1978) Flora of Mussoorie. Vol. 1. Bishen Singh \& Mahendra Pal Singh (eds.), Dehradun.

19. Sharma CM and Gairola S (2007) Prospects of carbon management in Uttarakhand: An overview. Samaj Vigyan Shodh Patrika (Special Issue-Uttarakhand-1), 23-34.

20. Sharma CM, Baduni NP, Gairola S, Ghildiyal SK and Suyal S (2010) The effect of slope aspects on the forest composition, community structure and soil nutrient status of some major natural temperate forest types of Garhwal Himalaya. J. For. Res. (In Press: to be published in 21(3)).

21. Sharma CM, Gairola S, Ghildiyal SK and Suyal S (2009c) Forest dependent livelihood in relation to socio-economic status of the people in temperate villages of Garhwal Himalaya: A case study. Mt. Res. Dev. 29(4), 308-319.

22. Sharma CM, Ghildiyal SK, Gairola S and Suyal S (2009b) Vegetation structure, composition and diversity in relation to the soil characteristics of temperate mixed broadleaved forest along an altitudinal gradient in Garhwal Himalaya. Ind. J. Sci. Tech. 2(7), 39-45.

23. Sharma CM, Suyal S, Gairola S and Ghildiyal SK (2009a) Species richness and diversity along an altitudinal gradient in moist temperate forest of Garhwal Himalaya. J. Am. Sci., 5(5), 119-128.

24. Sharma M and Dhaliwal DS (1997) Biological spectrum of the flora of Kullu district (Himachal Pradesh). J. Ind. Bot. Soc. 76, 283-284.

25. Singh JS (2006) Sustainable development of Indian Himalayan Region: Linking ecological and economic concern. Curr. Sci. 90(6), 784-788.

26. Uniyal SK, Awasthi A and Rawat GS (2002) Current status and distribution of commercially exploited medicinal and aromatic plants in upper Gori valley, Kumaun Himalaya, Uttaranchal. Curr. Sci. 82(10), 12461252.

27. Veblen T, Donoso C, Schlegel F and Escobar B (1981) Forest dynamics in southcentral Chile. J. Biogeog. 8, 211247.

28. Venu $P$ (2002) Some conceptual and practical issues in taxonomic research. Curr. Sci. 82(8), 924-933.

29. Villasenor J L, Maeda P, Rosell JA and Ortiz E (2007) Plant families as predictors of plant biodiversity in Mexico. Divers. Dist. 13, 871-876.

30. Young KR, Ulloa C, Luteyn JL and Knapp S (2002) Plant evolution and endemism in Andean South America: An introduction. Bot. Rev. 68, 4-21.
Research article

CIndian Society for Education and Environment (iSee)
"Phytodiversity of Garhwal Himalaya forest" http://www.indjst.org
Sarvesh Suyal et al. Indian J.Sci.Technol. 